\title{
Impurity-induced diffusion bias in epitaxial growth
}

\author{
Luís A. Nunes Amaral ${ }^{1}$ and Joachim Krug ${ }^{2}$ \\ 1 Theorie II, Institut für Festkörperforschung, Forschungszentrum Jülich, D-52425 Jülich, Germany \\ ${ }^{2}$ Fachbereich Physik, Universität-GH Essen, D-45117 Essen, Germany
}

(September 9, 1996)

\begin{abstract}
We introduce two models for the action of impurities in epitaxial growth. In the first, the interaction between the diffusing adatoms and the impurities is "barrier"-like and, in the second, it is "trap"-like. For the barrier model, we find a symmetry breaking effect that leads to an overall down-hill current. As expected, such a current produces Edwards-Wilkinson scaling. For the trap model, no symmetry breaking occurs and the scaling behavior appears to be of the conserved-KPZ type.
\end{abstract}

PACS numbers: 61.50.Cj, 05.40.+j, 68.35.Fx, 68.55Bd, 05.70.Ln, 68.55.-a, 68.35.Bs, 61.16.Fk

The effect of impurities on growth rate and morphology is a classic topic of crystal growth theory [1]. The most thoroughly studied case is the step flow growth of a vicinal surface, when the (immobile) impurities pin the advancing steps and thus lead to step bunching [2]3]. These theories are mesoscopic rather than microscopic in nature, in the sense that they describe the interaction of preexisting steps with discrete impurities [3] or an impurity concentration field [2.

The advent of modern crystal growth techniques aimed at manufacturing layers of atomic scale thickness, notably molecular beam epitaxy (MBE) [4], has lead to a renewed appreciation of the fact that small concentrations of impurity atoms on the growing surface can drastically influence the growth kinetics. A particularly striking aspect of these recent results is that the impurities may either lead to a deterioration of the growth quality - as would be expected according to the classic view [1] - or, conversely, they may play the role of surfactants in stabilizing smooth, layer-by-layer growth [5].

A clear example of the former type is the effect of hydrogen on the MBE of silicon [6,7]. It was observed that the presence of $\mathrm{H}$ in the growth chamber during $\mathrm{Si}$ deposition on $\mathrm{Si}(001)$ leads to a decrease of the epitaxial height [6], at which epitaxy breaks down and the growth becomes amorphous, proportional to the logarithm of the partial pressure of $\mathrm{H}$ [7]. The experiments ruled out the hypothesis that the breakdown of epitaxy might be due either to an increased coverage of $\mathrm{H}$ at the interface [6] or to its incorporation into the bulk [8]. Rather, it was concluded [7] that the hydrogen greatly speeds up the development of surface roughness due to a reduction of the diffusion length of Si adatoms 9.

The modification of the diffusion properties of the adatoms appears to be the most significant effect of the impurities also when they act as surfactants [10,11, though the nature of the modification - for example, whether the diffusion length is increased or decreased depends on the chemical species in a complicated way [5]. This sensitivity to atomic details is rather unexpected, and calls for the development of models which are more microscopic than previous approaches [2,3].

In this Letter, we introduce two models for the action of impurities in epitaxial growth. Rather than attempting a detailed description of some particular material, our aim is to define a 'minimal' model in which the consequences of the impurity-adatom interaction on the large scale morphology of the surface can be clearly elucidated. The study of oversimplified models [12,13] of 'ideal MBE' 14 has previously been very successful in clarifying the universality classes for kinetic roughening 14 16 and morphological instability 15,17,18 in the absence of impurities [19.

Our models reproduce the sensitive dependence on microscopic details mentioned above: Using two equally plausible microscopic interaction mechanisms of barrier-type and of trap-type, respectively - we find that for the barrier model the impurities neutralize the destabilizing effect of step edge barriers 15,18 and thus lead to smoother growth [20], while for the trap model the asymptotic morphology remains unaffected. In terms of the coarse-grained continuum description of the surface $[15,19$ we are able to trace the difference between the two models to the fact that the barriers modify the symmetry of the surface diffusion process, while the traps do not.

The models proposed in this Letter have three main ingredients (see Fig. 11).

i) Deposition and Diffusion: For simplicity, we consider a one-dimensional discrete substrate. Material is randomly deposited at a rate $F$. The deposition occurs in a solid-on-solid (SOS) manner, i.e. deposition at a position $i$ implies that the surface height $h(i)$ is increased by one unit. Every atom which has only one occupied neighbor (namely, in the layer below) is considered a mobile adatom which diffuses at a rate $D$; atoms with more bonds are immobile. When an adatom diffuses to a step edge from above we implement an additional energy barrier 21] by accepting a diffusion move down the step only with probability $p=\exp \left(-E_{S}\right)$, where the barrier energy $E_{S}$ is measured in units of $k_{B} T$. In the absence of impurities such step edge barriers are know to lead to unstable 
growth 15,18,19.

i)

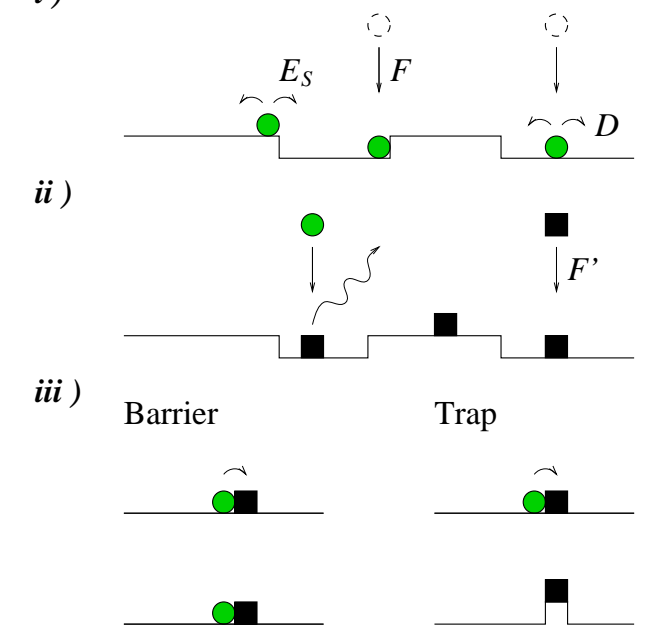

Fig. 1

FIG. 1. Schematic representation of the processes present in our models. Material is deposited at a rate $F$ and single atoms (gray circles) diffuse at a rate $D$. At step edges, adatom motion down the step is accepted with probability $p=\exp \left(-E_{S}\right)$, where $E_{S}$ is the step edge barrier. Impurities (squares) are deposited at a rate $F^{\prime}$ and are not allowed to diffuse. When an atom is deposited over an impurity, the latter evaporates and is replaced by the deposited atom. Impurities can interact with the adatoms through two distinct mechanism. In the barrier-like interaction, an adatom trying to diffuse on top of an impurity will have its move rejected. In the trap-like interaction, the same adatom would have been trapped, i.e., it would swap positions with the impurity and stop diffusing.

ii) Impurities: Based on the experimental observations described previously, we assume that there is a flux $F^{\prime}$ of impurities onto the growing surface. We restrict our study to the limit in which the diffusion rate of the impurities is much smaller than the diffusion rate of the adatoms, so that the impurities can be considered immobile. We also assume that impurities evaporate from the surface at a rate that keeps $\theta_{I}$, the impurity coverage, approximately constant. This is done by removing the impurity whenever a new atom is deposited on top of it. An important consequence of this rule is that the average lifetime of an impurity at a given site equals the monolayer deposition time.

iii) Interactions: Concerning the interactions between the impurities and the diffusing adatoms, we introduce two alternative models: the barrier and the trap model. In the barrier model, an adatom trying to diffuse onto a site occupied by an impurity will have its attempted move rejected. In the trap model, the same adatom will move on top of the impurity and then will swap positions. The end result is that it will no longer be able to diffuse because it has (at least) two "chemical bonds": to the atom below and to the impurity.

Fig. 2

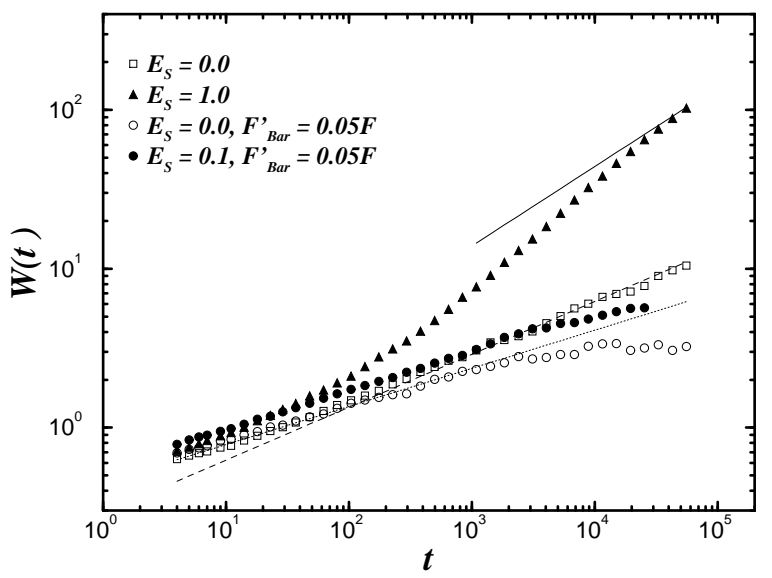

FIG. 2. Plot of the total surface width as a function of time (or coverage) for barrier-like impurities. The results shown were obtained for $L=1000$ and $D / F=10^{4}$. Averages were taken over 50 runs. In the absence of step edge barriers or impurities $W(t)$ diverges with an exponent $\beta \approx 1 / 3$. When step edge barriers are introduced, the instability in the growth process leads to an effective exponent close to one at intermediate times and the random deposition value $\beta \approx 0.5$ at long times. The presence of even a small amount of impurities leads to a significantly smoother surface. The lines are plotted as guides to the eye, and have slopes 0.24 (dotted), 0.33 (dashed), and 0.50 (full).

In our simulations we focus on the exponent $\beta$ describing the increase of the surface width 19] $W(t, L) \equiv$ $\left\langle(h-\langle h\rangle)^{2}\right\rangle^{1 / 2} \sim t^{\beta}$ in the early time regime $t \ll L^{z}$; here $L$ denotes the system size and the dynamic exponent is $z=1 /(1-2 \beta)$ for the class of (one-dimensional) models considered in this paper $[12$. To put the results into perspective, we will compare them to the predictions of the appropriate continuum equations for the coarse-grained height function $h(x, t)$; as usual, the average height $\langle h\rangle=F t$ will be subtracted. In the present context the following equation suffices 14 16]:

$$
\frac{\partial h}{\partial t}=\nu \nabla^{2} h-\frac{\lambda}{2} \nabla^{2}(\nabla h)^{2}-\kappa\left(\nabla^{2}\right)^{2} h+\eta,
$$

where the stochastic force $\eta(x, t)$ models the shot noise in the beam, and can be taken to be Gaussian with zero mean and covariance

$$
\left\langle\eta(x, t) \eta\left(x^{\prime}, t^{\prime}\right)\right\rangle=F \delta\left(x-x^{\prime}\right) \delta\left(t-t^{\prime}\right)
$$

The first term on the right hand side of (11) arises from the gradient expansion of an inclination-dependent, growth induced surface current 15,18. When it is present, it dominates the large scale morphology: For $\nu>0$ one obtains kinetic roughening of the Edwards-Wilkinson (EW) universality class 19,22 with $\beta=1 / 4$, while for $\nu<0$ 
Fig. 3(a)

the growth is unstable and a mound morphology is expected to develop [17, 19,23]. If, for reasons of symmetry (see below) $\nu=0$, the second, nonlinear term becomes important, and changes the roughening exponent to $\beta=1 / 3$ 15, 14, 16, (the "conserved Kardar-ParisiZhang" universality class [24). Finally, in many cases the growth-induced coefficients $\nu$ and $\lambda$ are small, and the early time behavior is dominated by the third term in (11), which arises from equilibrium surface diffusion 25] and leads to a (transient) value $\beta=3 / 8$ [12,13.

We consider first the case of a nonzero step edge barrier in the absence of impurities; then $\nu<0$ [15, 18 and one expects asymptotically unstable growth. As shown in Fig. 2, the instability sets in after an initial power law transient which terminates at about 100 monolayers. Later on, the destabilizing effect of the step edge barrier leads to wavelength selection and mound formation with a very rapid growth of the surface width. Once large slopes have appeared on the surface, there is hardly any transfer of matter between the different mounds and the exponent $\beta$ reaches the limiting value $\beta=1 / 2$ characteristic of random deposition 26.

When we introduce impurities of the barrier-type a striking change occurs. As is visually apparent from Fig. 2, the presence even of small amounts of barrier impurities leads to a significative decrease of the interface width. The exponent takes the value $\beta=0.24 \pm 0.03$, consistent with EW universality. The natural interpretation is that the impurities have caused the coefficient $\nu$ in Eq. (11) to change sign, from destabilizing $(\nu<0)$ to stabilizing $(\nu>0)$. The value of $\nu$ can be directly ascertained by measuring the average surface current for tilted substrates [18]. The results, shown in Figure 3(a), confirm our interpretation: Even a small flux of impurities $\left(F^{\prime} / F=0.05\right)$ leads to a sizable positive value of $\nu$, both in the presence of a step edge barrier and for $E_{S}=0$.

The reason for the change produced by the barrier impurities can be understood as follows. As is well known [15,18, the step edge barrier leads to an up-hill current because adatoms are rejected when trying to go down step edges and become integrated in the bulk when reaching an ascending step. This difference leads to an average current towards the up-step which destabilizes the surface, as described earlier. To visualize the effect of the impurities on this process, let us consider a step train moving from left to right; cf. Fig 3(b). An impurity can be deposited anywhere on a given terrace, so we can say that on average it is deposited in the middle between the two steps. However, as more material is deposited, the step edge to the left of the impurity advances towards it. On the other hand, the step edge to the right of the impurity moves away from it. Thus, the distance to the step to the left of the impurity is typically smaller than the distance to the right. Since the current away from the impurity on each side is proportional to the material deposited there (and thus to the length of that part of the terrace) we see that an average down-hill current is generated.
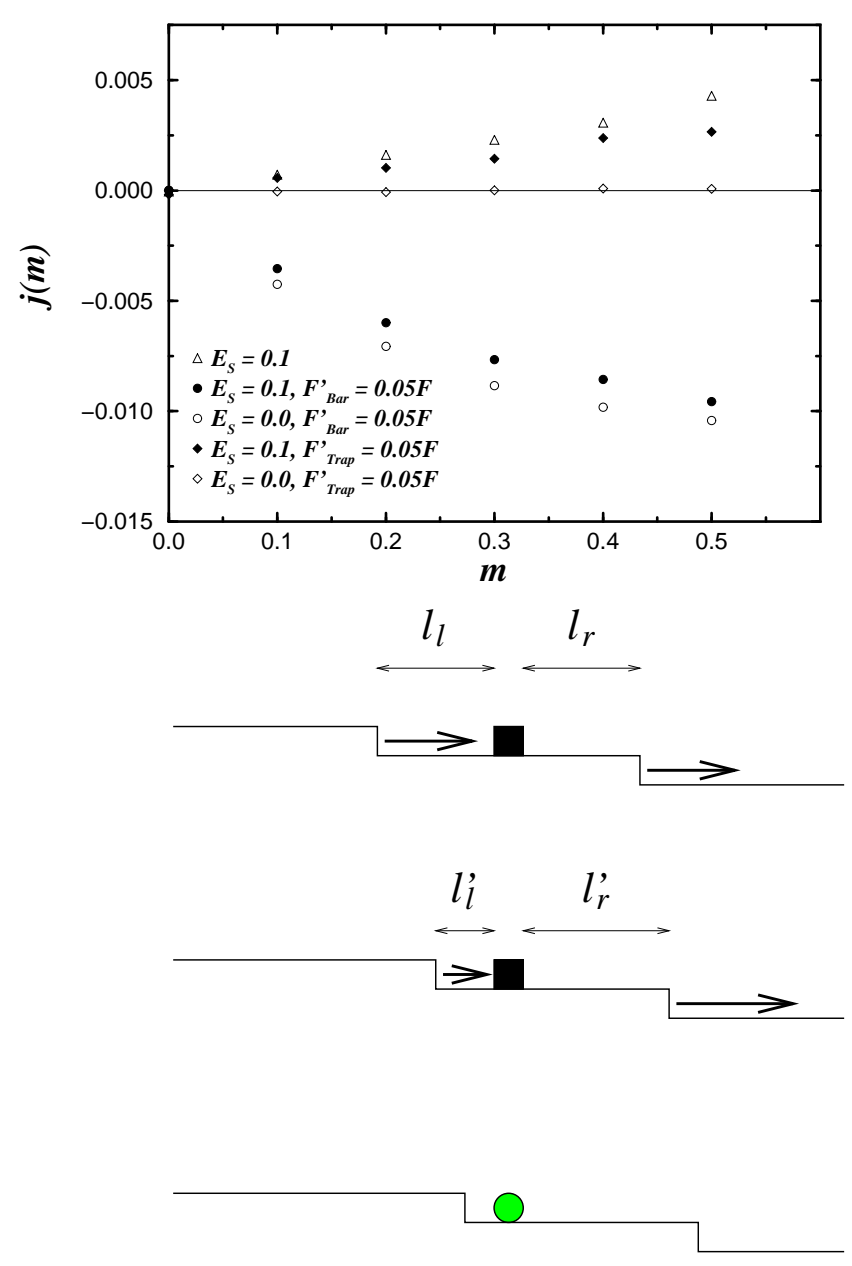

\section{Fig. 3(b)}

FIG. 3. a) Plot of the current $j(m)$ as a function of the tilt $m$ of the interface. The tilt is prescribed numerically through helicoidal boundary conditions. Our results make it clear that the barrier-like impurities lead to a negative current even in the presence of a step edge barrier, while the trap impurities leave the sign of the current unaffected. We used a smaller value of the step edge barrier because of the numerical difficulties in calculating the current (see Ref. [18]). b) Schematic representation of the effect of a barrier-like impurity on the motion of a step train. On average an impurity will be deposited on the middle of a terrace. However, while the upper step moves towards the impurity, the lower step moves away from it, so that in fact $l_{l}<l_{r}$, where $l_{l}\left(l_{r}\right)$ is the length of the portion of the step to the left (right) of the impurity. Since the up-hill current is proportional to $l_{l}$ and the down-hill current is proportional to $l_{r}$, we will have an average down-hill current which leads to a positive $\nu$ coefficient and a stable interface. Note that this mechanism can only be effective if during the time to deposit one monolayer the impurity will have been removed from its position; otherwise, the impurity would pin the step and destabilize the surface. 
In contrast, the trap impurities do not seem to significantly change the dynamics of the growth process, apart from an increase of the prefactor of the width (Fig. 4); certainly they are not able to suppress the destabilizing effect of the step edge barriers. This is confirmed by a measurement of the surface current, which remains uphill in the presence of traps, as shown in Fig. 3(a).

The traps do not induce a surface current because they cannot bias the diffusion of adatoms: The trap impurity makes itself felt only when the atom has already jumped onto it, and therefore no longer participates in the mass transport on the surface (when the impurity disappears due to the deposition of an additional atom, the trapped adatom remains immobile).

This point is brought out more clearly by considering the trap model without step edge barriers. For $E_{S}=0$, the pure model $\left(F^{\prime}=0\right)$ has a symmetry which forces $\nu=0$ in Eq. (11): For any local environment, the probability of a mobile adatom to jump to the right is equal to that for a jump to the left. Since this is true irrespective of the overall surface tilt, no growth-induced current can exist [27]. With $\nu=0$ the behavior of (1]) is dominated by the second, nonlinear term, and one expects $\beta=1 / 3$. Our simulations lead to a value of $\beta=0.33 \pm 0.03$, indicating that this symmetry is preserved by the trap impurities.

The increase in the prefactor of the width with increasing trap concentration can be interpreted as a decrease of the diffusion length $\ell_{D}$ : Indeed, it can be shown 28] that the prefactor scales as $\ell_{D}^{-4 d /(10+d)}$ for a $d$-dimensional surface. From the data shown in Fig. 4 we therefore estimate that an impurity flux $F^{\prime} / F=0.1$ decreases the diffusion length by almost a factor of three.

Fig. 4

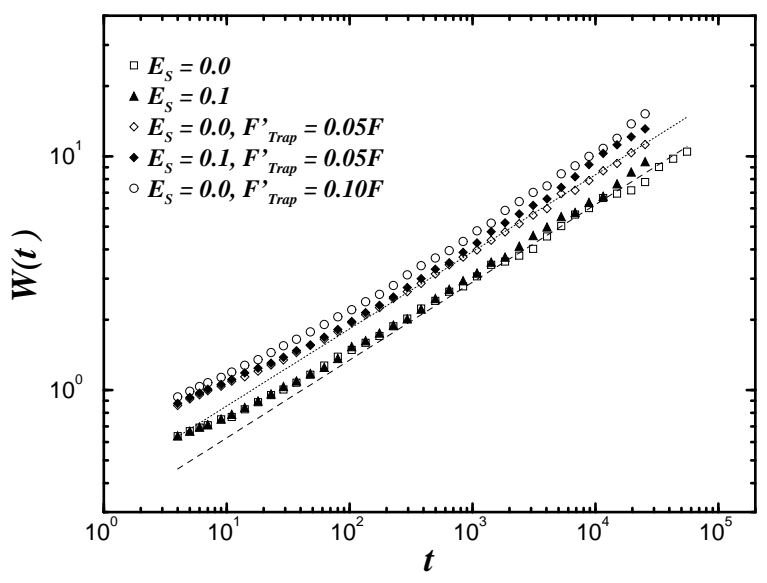

FIG. 4. Plot of the total surface width as a function of time for trap-like impurities, under the same conditions as in Fig. 2. In this case, the effect of the impurities is not as striking as for the barrier-like impurities. Even in the presence of step edge barriers, the main effect seems to be an increase in the prefactor of the width. The straight lines are plotted as guides to the eye, and have slope 0.33 .

Finally, one may ask why none of our models shows an impurity-induced growth instability of the kind considered in the classic theories of step bunching [2]3]. We believe that this is due to the fact that the lifetime of an impurity at a given position is, in our models, fixed to be of the order of the monolayer deposition time. In terms of the conventional step flow picture [1,2] it is evident that impurities can effectively pin steps only if they remain at a position much longer than the time required for a step to pass over a terrace. Thus, it appears important to consider models with a variable lifetime for the impurities.

We acknowledge stimulating discussions with M. Rost, M. Schimschak and P. Šmilauer.

[1] N. Cabrera and D. A. Vermilyea, in Growth and Perfection of Crystals, ed. by R. Doremus, B. Roberts and D. Turnbull (Wiley, New York 1958), p. 393.

[2] J. P. v.d. Eerden and H. Müller-Krumbhaar, Phys. Rev. Lett. 57, 2431 (1986).

[3] D. Kandel and J. D. Weeks, Phys. Rev. B 49, 5554 (1994).

[4] Molecular Beam Epitaxy, ed. by A. Cho, (AIP Press, Woodbury, NY 1994).

[5] B. Voigtländer, A. Zinner, T. Weber and H. P. Bonzel, Phys. Rev. B 51, 7583 (1995), and references therein.

[6] D. J. Eaglesham, J. Appl. Phys. 77, 3597 (1995).

[7] D. P. Adams, S. M. Yalisove, and D. J. Eaglesham, Appl. Phys. Lett. 63, 3571 (1993).

[8] M. Copel and R. M. Tromp, Phys. Rev. Lett. 72, 1236 (1994).

[9] J. E. Vasek, Z. Zhang, C. T. Salling, and M. G. Lagally, Phys. Rev. B 51, 17207 (1995).

[10] Z. Zhang and M. G. Lagally, Phys. Rev. Lett. 72, 693 (1994).

[11] D. Kandel and E. Kaxiras, Phys. Rev. Lett. 75, 2742 (1995).

[12] D. E. Wolf and J. Villain, Europhys. Lett. 13, 389 (1990).

[13] S. Das Sarma and P. Tamborenea, Phys. Rev. Lett. 66, 325 (1991); P. I. Tamborenea and S. Das Sarma, Phys. Rev. E 48, 2575 (1993).

[14] Z.-W. Lai and S. Das Sarma, Phys. Rev. Lett. 66, 2348 (1991).

[15] J. Villain, J. Phys. France I 1, 19 (1991).

[16] L.-H. Tang and T. Nattermann, Phys. Rev. Lett. 66, 2899 (1991).

[17] M. Siegert and M. Plischke, Phys. Rev. Lett. 68, 2035 (1992); Phys. Rev. E 50, 917 (1994).

[18] J. Krug, M. Siegert, and M. Plischke, Phys. Rev. Lett. 70, 3271 (1993).

[19] For reviews see A.-L. Barabási and H. E. Stanley, Fractal Concepts in Surface Growth (Cambridge University Press, Cambridge, 1995); J. Krug, Adv. Phys. (in press).

[20] A related mechanism has been described by I. Markov, Phys. Rev. B 50, 11271 (1994).

[21] G. Ehrlich and F. G. Hudda, J. Chem. Phys. 44, 1039 
(1966); R. L. Schwoebel and E. J. Shipsey, J. Appl. Phys. 37, 3682 (1966).

[22] S. F. Edwards and D. R. Wilkinson, Proc. R. Soc. Lond. A381, 17 (1982).

[23] P. Šmilauer and D. D. Vvedensky, Phys. Rev. B 52, 14263 (1995).

[24] M. Kardar, G. Parisi, and Y.-C. Zhang, Phys. Rev. Lett. 56, 889 (1986).
[25] W. W. Mullins, J. Appl. Phys. 28, 333 (1957).

[26] C. J. Lanczycki and S. Das Sarma, Phys. Rev. Lett. 76, 780 (1996).

[27] For related symmetries in other models see J.Krug in Ref. 19 .

[28] L. Brendel, H. Kallabis, J. Krug, M. Schroeder, J. Villain and D.E. Wolf (unpublished); see also J. Krug in Ref. 《19. 